\title{
OSCILLATION PROPERTIES OF EVEN-ORDER LINEAR DIFFERENTIAL EQUATIONS
}

\author{
BY \\ ROBERT W. HUNT $\left(^{1}\right)$
}

1. Introduction. The ordinary, self-adjoint differential equation

$$
\left[r(x) y^{(n)}\right]^{(n)}+p(x) y=0
$$

is considered for $r(x)$ and $p(x)$ continuous and $r(x)>0, p(x) \neq 0$ on $[a, \infty)$. For $n=1$, this equation has been the subject of many investigations over a number of years. For $n=2$, J. H. Barrett [1]-[3] and W. Leighton and Z. Nehari [7] have recently considered equations of this type. For arbitrary $n$, similar equations have been investigated by W. T. Reid [8], by $H$. Kaufman [6], by H. M. and R. L. Sternberg [6], [9], and by the author [5].

In the present paper, a general boundary problem is considered for a solution $y(x)$ of $(\mathrm{A})$; namely,

$$
\begin{aligned}
& y(a)=y^{\prime}(a)=\cdots=y^{(n-1)}(a)=y_{1}(a)=\cdots=y_{1}^{(n-i-1)}(a)=0, \\
& y(b)=y^{\prime}(b)=\cdots=y^{(i-1)}(b)=0
\end{aligned}
$$

for $1 \leqq i \leqq n, b>a$, and $y_{1}(x) \equiv r(x) y^{(n)}(x)$. If $y(x)$ satisfies the first of these conditions, then $y(x)$ is said to be a solution of (A) with a zero of order $2 n-i$ at $x=a$, i.e., after the $(n-1)$ st derivative of $y(x)$, derivatives of $y_{1}(x)$ are used. For $i=n$, this is similar to boundary problems considered in [1]-[9]. In general, however, the techniques utilized in these latter investigations are dependent on the fact that $i=n$. In $\$ 2$, a basic property of zeros of solutions of (A) is established. In $\$ \$ 3$ and 4 , separation and oscillation properties of solutions of (A) are established for $p(x)>0$ on $[a, \infty)$.

2. A basic property of zeros of solutions of (A). Consider the equations

$$
\begin{aligned}
& {\left[r(x) y^{(n)}\right]^{(n)}+p(x) y=0,} \\
& {\left[r(x) y^{(n)}\right]^{(n)}-p(x) y=0,}
\end{aligned}
$$

both of which have $r(x)$ and $p(x)$ continuous and positive on $[a, \infty)$. An

Presented to the Society, January 24, 1963; received by the editors December 2, 1963.

( ${ }^{1}$ This paper is part of a thesis submitted to the faculty of the University of Utah in partial fulfillment of the requirements for the degree of Doctor of Philosophy. The author expresses thanks to the thesis director, Professor John H. Barrett. 
important general property of zeros of solutions of (1) and (2) is then contained in the following theorem.

TheOREM 1. If $i$ is an even [odd] positive integer and $y(x)$ is a nontrivial solution of (1) $[(2)]$ with a zero of order $\geqq 2 n-i$ at $x=a$, then $y(x)$ cannot have a zero of order $\geqq i$ on $(a, \infty)$, i.e., there does not exist a nontrivial solution satisfying the conditions (B).

Proof. Suppose that, for $i \leqq n, y(x)$ has a zero of order $2 n-i$ at $x=a$ and has a zero of order $i$ at $x=b$ on $(a, \infty)$ and that $y(x)$ has $m$ zeros on $(a, b), m \geqq 0$. Let $c \in(a, b]$ be the first zero of $y(x)$ on $(a, b]$. It shall then first be established that $y_{1}^{(n-1)}(x)$ must have a zero on $(a, c]$, regardless of whether $i$ is even or odd.

Note that $y(a)=y^{\prime}(a)=\ldots=y_{1}(a)=y_{1}^{\prime}(a)=\ldots=y_{1}^{(n-i-1)}(a)=0$ (where, if $i=n, y_{1}^{(-1)}(a)$ is interpreted as $y^{(n-1)}(a)$ ) and $y(b)=y^{\prime}(b)=\ldots$ $=y^{(i-1)}(b)=0$. Then successive application of Rolle's Theorem to $y(x)$, $y^{\prime}(x), \cdots, y^{(i)}(x)$ gives that $y^{(i)}(x)$ has at least $m+i$ zeros on $(a, b)$, a zero of order $2(n-i)$ at $x 2 a$, and does not vanish at $x=b$. Continuation of the process shows that $y_{1}(x)$ has at least $m+i$ zeros on $(a, b)$, a zero of order $n-i$ at $x=a$, and does not vanish at $x=b$. Then finally, $y_{1}^{(n-1)}(x)$ must have at least $m+1$ zeros on $(a, b)$. Suppose that $y_{1}^{(n-1)}(x) \neq 0$ on $(a, c]$. Then $y_{1}^{(n-1)}(x)$ has at least $m+1$ zeros on $(c, b)$ and hence $y_{1}^{(n)}(x)$ has at least $m$ zeros on $(c, b)$. But $y_{1}^{(n)}(x)= \pm p(x) y(x)$ and thus $y(x)$ would have $m$ zeros on $(c, b)$, a contradiction. (If $m=0$, the last three statements are not necessary.) Note that $y_{1}^{(n-1)}(x)$ has exactly $m+1$ zeros on $(a, b)$.

Now let $x=x_{0}$ be the first zero of $y_{1}^{(n-1)}(x)$ on $(a, c]$ and assume, with no loss of generality, that $y(x)>0$ on $(a, c)$. Then $y_{1}^{(n-i)}(a) \geqq 0$ regardless of whether $i$ is even or odd. From (1)

$$
y_{1}^{(n-1)}\left(x_{0}\right)-y_{1}^{(n-1)}(a)=-y_{1}^{(n-1)}(a)=-\int_{a}^{x_{0}} p(x) y d x<0,
$$

and $y_{1}^{(n-1)}(a)>0$. Similarly, using $(2), y_{1}^{(n-1)}(a)<0$. If $i=1$, the last statement is a contradiction and the special case of the theorem for $i=1$ and equation (2) is established.

Suppose $i>1$ and note that $y_{1}^{(n-2)}(x)$ must have a zero on $\left(a, x_{0}\right)$ since the $m+1$ zeros of $y_{1}^{(n-1)}(x)$ fall between the extreme zeros of $y_{1}^{(n-2)}(x)$ on $(a, b)$. This comes from the first part of the proof. Also, $y_{1}^{(n-2)}(x)$ has exactly $m+2$ zeros on $(a, b)$. Similarly, $y_{1}^{(n-3)}(x)$ must have a zero before the first zero of $y_{1}^{(n-2)}(x)$ on $\left(a, x_{0}\right)$; and $y_{1}^{(n-3)}(x)$ has exactly $m+3$ zeros on $(a, b)$. This same type of reasoning can then be followed to establish that $y_{1}^{(n-i)}(x)$ must have a zero on $\left(a, x_{0}\right)$ before the first zero on $\left(a, x_{0}\right)$ of $y_{1}^{(n-i+1)}(x)$ and 
$y^{(n-i)}(x)$ has exactly $m+i$ zeros on $(a, b)$. If $i=1$, of course, this entire procedure consists only of the statement that $y^{(n-1)}(x)$ vanishes at $x_{0}$ on $(a, c]$.

Then, using (1), $y_{1}^{(n-2)}(x)$ must begin at $x=a$ with positive slope and have a zero before its derivative has a zero. This is possible only if $y_{1}^{(n-2)}(a)<0$. Then $y_{1}^{(n-3)}(x)$ must begin at $x=a$ with negative slope and have a zero before its derivative does so, implying that $y_{1}^{(n-3)}(a)>0$. Successive applications of this argument yield the fact that $y_{1}^{(n-i)}(a)<0$ if $i$ is an even integer. This is a contradiction since $y_{1}^{(n-i)}(a)>0$. Hence, the portion of the theorem regarding (1) and even values of $i$ is established. In an analogous way, using (2), $y_{1}^{(n-i)}(a)<0$ if $i$ is odd; and a contradiction results.

The theorem is now proved for $i \leqq n$. For $i>n$, it suffices to note that the change of variable $x=a-t+b$ leaves (1) and (2) unchanged while transforming $x=a$ into $t=b$ and $x=b$ into $t=a$. Thus, a solution $y(x)$ with a zero of order $2 n-i$ at $x=a$ and order $i$ at $x=b$ is transformed into a solution with a zero of order $i$ at $t=a$ and order $2 n-i$ at $t=b$. Then the case $i>n$ can be taken care of by the above proof. This completes the proof of the theorem.

Included in Theorem 1 is a generalization of Lemma 8.2 in [7, p. 358], which states that no nontrivial solution of (1) for $n=2$ has more than one double zero (i.e., a zero of order two). Theorem 1 includes the fact that no nontrivial solution of (1) has more than one $n$ th-order zero. In fact, using the following definition, the following corollary holds.

Definition. The number $\eta_{1}(a)$ is the smallest number $b$ on $(a, \infty)$ such that the boundary conditions

$$
y(a)=y^{\prime}(a)=\cdots=y^{(n-1)}(a)=0=y(b)=y^{\prime}(b)=\cdots=y^{(n-1)}(b)
$$

are satisfied nontrivially by a solution $y(x)$ of (1) or (2). This is the type of boundary problem considered in $[1],[3],[5],[7],[8],[9]$.

Corollary 1. If $n$ is even [odd], then $\eta_{1}(a)$ does not exist for (1) [(2)].

3. Separation properties of solutions of (1). First of all, Theorem 1 can be utilized to give the following generalization of Lemma 9.1 in $[7$, p. 361].

TheOREM 2. If $y(x)$ and $z(x)$ are linearly independent solutions of (1) which have zeros of order $\geqq 2 n-2$ at $x=a$, then the zeros of $y(x)$ and $z(x)$ separate each other on $(a, \infty)$.

Proof. If $y(x)$ has consecutive zeros between which $z(x)$ has no zero, a basic result contained in [7, Lemma 1.2, p. 327] gives the existence of a constant $k$ such that $y(x)-k z(x)$ has a double zero in the interval between the zeros of $y(x)$ under consideration. But $y(x)-k z(x)$ is a solution of (1) with a zero of order $2 n-2$ at $x=a$ and cannot have a zero of order two 
on $(a, \infty)$ by Theorem 1 . Also, $y(x)$ and $z(x)$ cannot have a zero in common for then a constant $k$ could be chosen so that $y(x)-k z(x)$ has a double zero at the common zero, a contradiction. Therefore, the zeros of $y(x)$ and $z(x)$ must separate each other on $(a, \infty)$.

Let $\left\{u_{i}(x)\right\}(i=1,2,3, \cdots, n)$ be a set of solutions of (1) which satisfy the boundary conditions

$$
\begin{gathered}
{\left[u_{i}(x)\right]^{(j-1)}=0 \text { for } x=a \text { and } i, j=1,2, \cdots, n,} \\
{\left[u_{i, 1}(x)\right]^{(j-1)}=\delta_{i j} \quad \text { for } x=a \text { and } i, j=1,2, \cdots, n} \\
\text { and } u_{i, 1}(x)=r(x) u_{i}^{(n)}(x) .
\end{gathered}
$$

Also, let $\left\{U_{i}(x)\right\}(i=1,2, \cdots, n)$ be a set of solutions of (1) satisfying the conditions

$$
\begin{aligned}
{\left[U_{i}(x)\right]^{(-1)}=} & \delta_{i j} \text { for } x=a \text { and } i, j=1,2, \cdots, n, \\
{\left[U_{i, 1}(x)\right]^{(j-1)}=} & 0 \text { for } x=a \text { and } i, j=1,2, \cdots, n \\
& \text { and } U_{i, 1}(x)=r(x) U_{i}^{(n)}(x) .
\end{aligned}
$$

Theorem 3. Consider the sets $\left\{u_{i}(x)\right\}$ and $\left\{U_{i}(x)\right\}(i=1,2, \cdots, n)$ of solutions of (1) satisfying the conditions of (4) and (5), respectively. Then the zeros of $u_{n}(x)$ and the zeros of any member of the set $\left\{U_{i}(x)\right\}(i=1,2, \cdots, n)$. or of $\left\{u_{i}(x)\right\}(i=1,2, \cdots, n-1)$, separate each other on $(a, \infty)$.

Proof. The proof uses similar techniques to that of Theorem 1. Consider first, the set $\left\{U_{i}(x)\right\}(i=1,2, \cdots, n)$, and let $U_{i}(x)$ be one of the member: of the set and $y_{i}(x)$ any linear combination of $U_{i}(x)$ and $u_{n}(x)$. If it car be established that $y_{i}(x)$ cannot have a double zero on $(a, \infty)$, it will follow as in the proof of Theorem 2 that the zeros of $u_{n}(x)$ and $U_{i}(x)$ must separat. each other. Thus, suppose that $y_{i}(x)$ does have a double zero on $(a, \infty)$ a $x=b$ and that $y_{i}(x)$ has $m$ zeros on $(a, b), m \geqq 0$. Also, let $x=c$ on $(a, b$ be the first of these zeros to the right of $x=a$. It will first be establisher that, under these assumptions, $y_{i, 1}^{(n-1)}(x)$ must have a zero on $(a, c]$. Reca $y_{i, 1}(x)=r(x) y_{i}^{(n)}(x)$.

Successive applications of Rolle's Theorem to $y_{i}(x), y_{i}^{\prime}(x), \cdots, y_{i}^{(i-1)}(\lambda$ show that $y_{i}^{(i)}(x)$ must have at least $m+1$ zeros on $(a, b)$. Then, similarl! $y_{i, 1}^{(n-1)}(x)$ must have at least $m+1$ zeros on $(a, b)$. Suppose $y_{i, 1}^{(n-1)}(x)$ dor not have a zero on $(a, c]$. Then it has $m+1$ zeros on $(c, b)$ and hence $y_{i, 1}^{(n)}(2$ or $y_{i}(x)$, has $m$ zeros on $(c, b)$, a contradiction. Note that if $m=0$ and hen $b=c$, the latter part of the argument is unnecessary. In any case, it follov that $y_{i, 1}^{(n-1)}(x)$ has a zero on $(a, c]$. Note that $y_{i, 1}^{(n-1)}(x)$ has exactly $m+$ zeros on $(a, b)$. Note, also, from the above argument that $y_{i}^{(i)}(x)$ must ha 
exactly $m+1$ zeros on $(a, b)$ and that these zeros lie between the first and last zeros of $y_{i}^{(i-1)}(x)$ on $(a, b)$.

Now, let $x=x_{0}$ be the first zero of $y_{i, 1}^{(n-1)}(x)$ on $(a, c]$ and assume, with no loss of generality, that $y(x)>0$ on $(a, c)$. This implies that $y^{(i-1)}(a)>0$. Then

$$
y_{i, 1}^{(n-1)}\left(x_{0}\right)-y_{i, 1}^{(n-1)}(a)=-y_{i, 1}^{(n-1)}(a)=-\int_{a}^{x_{0}} p(x) y d x<0,
$$

and $y_{i, 1}^{(n-1)}(a)>0$. Thus $y_{i, 1}^{(n-2)}(x)$ must be zero and have positive slope at $x=a$ which implies that $y_{i, 1}^{(n-2)}(x)>0$ in a right neighborhood of $x=a$. Next, $y_{i, 1}^{(n-3)}(x)$ must be zero and have zero slope at $x=a$, with positive slope in a right neighborhood of $x=a$, so that $y_{i, 1}^{(n-3)}(x)>0$ in a right neighborhood of $x=a$. This same property must hold for the succeeding functions $y_{i, 1}^{(n-4)}(x), \cdots, y_{i}^{(i)}(x)$. Then $y^{(i-1)}(x)$ must begin at $x=a$ with positive slope and have positive slope in a right neighborhood of $x=a$. Furthermore, by the comment following the first part of the proof, $y^{(i-1)}(x)$ must have a zero before its derivative $y^{(i)}(x)$ has a zero on $(a, b)$. These properties are possible only if $y^{(i-1)}(a)<0$, a contradiction. Thus, the zeros of $u_{n}(x)$ and those of any member of the set $\left\{U_{i}(x)\right\}(i=1,2, \cdots, n)$, separate each other.

The proof of the latter part of the theorem follows the same lines as that above. Using the same notation, the proof that $y_{i, 1}^{(n-1)}(x)$ must have a zero on $(a, c]$ is obtained as before by successive applications of Rolle's Theorem. Hence, in this case, $y_{i, 1}^{(i)}(x)$ has at least $m+1$ zeros on $(a, b)$; and, furthermore, $y_{i, 1}^{(n-1)}(x)$ has at least $m+1$ zeros on $(a, b)$. If $y_{i, 1}^{(n-1)}(x)$ did not have a zero on $(a, c]$, a contradiction would result as before. Also, $y_{i, 1}^{(i)}(x)$ must have exactly $m+1$ zeros on $(a, b)$ and these lie between the first and last zeros of $y_{i, 1}^{(i-1)}(x)$ on $(a, b)$.

Finally, as before, it follows that upon assuming $y(x)>0$ on $(a, c)$, and hence that $y_{i, 1}^{(i-1)}(a)>0, y_{i, 1}^{(i-1)}(x)$ must have slope zero at $x=a$ and positive slope in a right neighborhood of $x=a$. Furthermore, $y_{i, 1}^{(i-1)}(x)$ has a zero before its derivative has a zero. This gives $y_{i, 1}^{(i-1)}(a)<0$, a contradiction. This completes the proof of the theorem.

4. Conditions for oscillation of solutions of (1). The main result of this section is contained in the following theorem.

Theorem 4. Suppose that

$$
\int^{\infty}\left({ }^{x} I^{n} \frac{t^{n-2}}{r}\right) p(x) d x=\infty
$$

and

$$
\int^{\infty}\left(I^{n} \frac{p}{r(x)}\right) d x=\infty
$$


(where ${ }^{x} I^{n}$ denotes the nth iterated integral). Then there exists a set of $2 n$ linearly independent, oscillatory solutions. (infinitely many zeros on $[a, \infty)$ ) of (1) with one oscillatory solution whose zeros separate the zeros of each of the other oscillatory solutions $(n>1)$.

Proof. Assume that the theorem is false, and consider the solution $y(x)$ $\equiv u_{n}(x)$ with a zero of order $2 n-1$ at $x=a$. Let $x=b$ be the last zero of $y(x)$ on $[a, \infty)$, and let $m$ be the number of zeros of $y(x)$ on $(a, \infty), m \geqq 0$. All zeros of $y(x)$ on $(a, \infty)$ must be simple by Theorem 1 . Assume, with no loss of generality, that $y(x)>0$ on $(b, \infty)$. Then if $m$ is even, $y(x)>0$ between $x=a$ and the first zero of $y(x)$; if $m$ is odd, $y(x)<0$ between $x=a$ and the first zero of $y(x)$.

By repeated application of Rolle's Theorem, it follows that in each interval between consecutive zeros of $y(x)$ there exists exactly one simple zero (in order, right to left) of each of the functions $y^{\prime}(x), y^{\prime \prime}(x), \cdots, y_{1}(x), y_{1}^{\prime}(x)$, $\cdots, y_{1}^{(n-1)}(x)$; and none of these functions have zeros coinciding with those of $y(x)$. If one of these functions had more than one zero on such an interval or a zero coinciding with one of $y(x)$, further application of Rolle's Theorem would show that $y_{1}^{(n)}(x)=-p(x) y(x)$ had a zero on that interval, an impossibility. Furthermore, $y^{\prime}(x), y^{\prime \prime}(x), \cdots, y_{1}(x), y_{1}^{\prime}(x), \cdots, y_{1}^{(n-1)}(x)$ are all positive for $x=b$ (unless $b=a$ in which case the functions are all positive in a right neighborhood of $x=b=a$ ) since they are all positive in a right neighborhood of $x=a$ for $m$ even and all negative in a right neighborhood of $x=a$ for $m$ odd and all vanish $m$ times on $(a, b)$.

It will now be shown that $y^{\prime}(x), y^{\prime \prime}(x), \cdots, y_{1}(x), \cdots, y_{1}^{(n-2)}(x)$ do not have any zeros at all on $(b, \infty)$ and hence are all positive on $(b, \infty)$. Assume that $y^{\prime}(x)$ has a zero at $x=c$ on $(b, \infty)$. Then, by repeated use of Rolle's Theorem, $y^{\prime \prime}(x), y^{\prime \prime \prime}(x), \cdots, y_{1}(x), \cdots, y_{1}^{(n-1)}(x)$ all have zeros on $(b, c)$ and are thus all negative on $(c, \infty)$, as is $y^{\prime}(x)$, since another zero of any of these functions on $(c, \infty)$ would lead to another zero of $y(x)$ on $(b, \infty)$. But $y(x)$ cannot remain positive on $(c, \infty)$ if $y^{\prime}(x)^{\prime}$ and $y^{\prime \prime}(x)$ are negative on that interval and hence a contradiction results. By the same reasoning, it follows that $y^{\prime \prime}(x), y^{\prime \prime \prime}(x), \cdots, y^{(n-1)}(x)$ cannot have zeros on $(b, \infty)$.

Next, suppose that $y_{1}(x)$ has a zero at $x=c$ on $(b, \infty)$. Then $y_{1}^{\prime}(x), y_{1}^{\prime \prime}(x)$, $\cdots, y_{1}^{(n-1)}(x)$ must all have zeros on $(b, c)$ and remain negative on $(c, \infty)$ and $y_{1}(x)$ is negative on $(c, \infty)$. Now

$$
\begin{aligned}
y^{(n-1)}(x) & =y^{(n-1)}(c)+\int_{c}^{x} y^{(n)}(t) d t \\
& =y^{(n-1)}(c)+\int_{c}^{x} \frac{y_{1}(t)}{r(t)} d t .
\end{aligned}
$$

Since $y(x)$ is an increasing function on $(c, \infty)$ and $y_{1}^{(n-1)}(c)<0$, it follows that 


$$
\begin{aligned}
y_{1}^{(n-1)}(x) & =y_{1}^{(n-1)}(c)-\int_{c}^{x} p(t) y(t) d t \\
& <-\int_{c}^{x} p(t) y(t) d t<-y(c) \int_{c}^{x} p(t) d t .
\end{aligned}
$$

Further integrations and the conditions on $y_{1}(x), y_{1}^{\prime}(x), \cdots, y_{1}^{(n-2)}(x)$ at $x=c$ thus yield

$$
y_{1}(x)<-y(c){ }_{c}^{x} I^{n} p,
$$

where ${ }_{c}^{x} I^{n} p$ is the $n$th iterated integral of $p(x)$.

Then

$$
\begin{aligned}
y^{(n-1)}(x) & =y^{(n-1)}(c)+\int_{c}^{x} \frac{y_{1}(t)}{r(t)} d t \\
& <y^{(n-1)}(c)-y(c) \int_{c}^{x} \frac{\left({ }_{c}^{t} I^{n} p\right)}{r(t)} d t
\end{aligned}
$$

and, using the hypothesis, $y^{(n-1)}(x) \rightarrow-\infty$ as $x \rightarrow \infty$. Then $y^{(n-1)}(x)$ must have a zero on $(c, \infty)$, a contradiction. By the same argument as used for $y^{\prime}(x), y^{\prime \prime}(x), \cdots, y^{(n-1)}(x)$, it now follows that $y_{1}^{\prime}(x), y_{1}^{\prime \prime}(x), \cdots, y_{1}^{(n-2)}(x)$ cannot have zeros on $(b, \infty)$.

Now $y_{1}^{(n)}(x)=-p(x) y(x)$ so that $y_{1}^{(n)}(x)$ is negative on $(b, \infty)$ and hence $y_{1}^{(n-1)}(x)$ is a decreasing function on $(b, \infty)$ and can have at most one zero on $(b, \infty)$. Assume that $y_{1}^{(n-1)}(x)$ has no zeros on $(b, \infty)$. Note that $y_{1}(x)$ $\geqq x^{n-2}$ on $\left(x_{1}, \infty\right)$ for some $x_{1} \geqq b$ since $y_{1}, y_{1}^{\prime}, \cdots, y_{1}^{(n-1)}$ are all positive functions on $(b, \infty)$. Furthermore,

$$
y^{(n-1)}(x)=y^{(n-1)}(b)+\int_{b}^{x} \frac{y_{1}(t)}{r(t)} d t>\int_{x_{1}}^{x} \frac{t^{n-2}}{r(t)} d t
$$

Then, because of the conditions on $y(x), y^{\prime}(x), \cdots, y^{(n-2)}(x)$ at $x=x_{1}, n-1$ integrations give

$$
y(x)>_{x_{1}}^{x} I^{n} \frac{t^{n-2}}{r(t)}
$$

From these results,

$$
\begin{aligned}
\int_{x_{1}}^{\infty} y_{1}^{(n)}(x) d x & =\lim _{x \rightarrow \infty} y_{1}^{(n-1)}(x)-y_{1}^{(n-1)}\left(x_{1}\right) \\
& =-\int_{x_{1}}^{\infty} p(x) y(x) d x \leqq-\int_{x_{1}}^{\infty}\left({ }^{x} I^{n} \frac{t^{n-2}}{r(t)}\right) p(x) d x=-\infty,
\end{aligned}
$$

and hence $y_{1}^{(n-1)}(x)$ does have a zero for some value $x=c$ on $\left(x_{1}, \infty\right)$. But then $y_{1}^{(n-2)}(x)$ is positive on $(c, \infty)$ while $y_{1}^{(n-1)}(x)$ and $y_{1}^{(n)}(x)$ are negative on $(c, \infty)$, an impossibility. Thus, $y(x) \equiv u_{n}(x)$ cannot have a "last" zero on $(a, \infty)$ and must be oscillatory. From Theorem 3 , the zeros of $u_{n}(x)$ separate those of any member of the sets $\left\{U_{i}(x)\right\}(i=1,2, \cdots, n)$, and 
$\left\{u_{i}(x)\right\}(i=1,2, \cdots, n-1)$; and thus each of these solutions of (1) must oscillate. This completes the proof of the theorem.

Corollary 2. If $\int^{\infty} x^{2 n-2} p(x) d x=\infty$ and $\int^{\infty}\left(x^{n-1} / r(x)\right) d x=\infty$, then there exists a set of $2 n$ linearly independent solutions of (1) as in Theorem 4.

Proof. $\int^{\infty}\left({ }^{x} I^{n}\left(t^{n-2} / r(t)\right)\right) p(x) d x=\infty$ implies $\int^{\infty} x^{2 n-2} p(x) d x=\infty$ and $\int^{\infty}\left({ }^{x} I^{n} p / r(t)\right) d x=\infty$ implies that $\int^{\infty}\left(x^{n-1} / r(x)\right) d x=\infty$. The result of the corollary then follows.

For $n=2$, the following corollary generalizes Theorem 11.4 of [7] which Leighton and Nehari have for the case $r(x) \equiv 1$.

Corollary 3. If $\int^{\infty}\left({ }^{x} I^{2} r\right) p(x) d x=\infty$ and $\int^{\infty}\left(\left({ }^{x} I^{2} p\right) / r(x)\right) d x=\infty$, then (1) (for $n=2)$ is oscillatory.

Proof. By Corollary 9.10 of [7], the solutions of (1) for $n=2$ are either all oscillatory or all nonoscillatory. Application of Theorem 4 then proves the desired result.

Corollary 4. If $\int^{\infty}\left({ }^{x} I^{n}\left(t^{n-2} / r(t)\right)\right) p(x) d x=\infty$ and $\int^{\infty}\left({ }^{x} I^{n} p\right) / r(x) d x$ $=\infty$, then any solution of (1) with a zero of order $2 n-2$ at $x=a$ is oscillatory.

Proof. Theorem 2 and Theorem 4 combine to give this result.

\section{BIBLIOGRAPHY}

1. J. H. Barrett, Disconjugacy of a self-adjoint differential equation of the fourth order, Pacific J. Math. 11 (1961), 25-37.

2. , Systems-disconjugacy of a fourth-order differential equation, Proc. Amer. Math. Soc. 12 (1961), 205-213.

3. self-adjoint differential equations and second-order matrix differential systems, Canad. J. Math. 13 (1961), 625-638.

4. E. A. Coddington and N. Levinson, Theory of ordinary differential equations, McGrawHill, New York, 1955.

5. R. W. Hunt, The behavior of solutions of ordinary, self-adjoint differential equations of arbitrary even order, Pacific J. Math. 12 (1962), 945-961.

6. H. Kaufman and R. L. Sternberg, $A$ two-point boundary problem for ordinary self-adjoint differential equations of even order, Duke Math. J. 20 (1953), 527-531.

7. Walter Leighton and Z. Nehari, On the oscillation of solutions of self-adjoint linear differential equations of the fourth order, Trans. Amer. Math. Soc. 89 (1958), 325-377.

8. W. T. Reid, Oscillation criteria for self-adjoint differential systems, Trans. Amer. Math. Soc. 101 (1961), 91-106.

9. H. M. Sternberg and R. L. Sternberg, A two-point boundary problem for ordinary selfadjoint differential equations of fourth order, Canad. J. Math. 6 (1954), 416-419.

SOUTHERN ILLINOIS UNIVERSITY,

Carbondale, Illinois 